\title{
PICK INTERPOLATION FOR A UNIFORM ALGEBRA
}

\author{
J. WERMER
}

Department of Mathematics, Brown University

Providence, Rhode Island 02912-0001, U.S.A.

I am reporting on joint work with Brian Cole and Keith Lewis.

Let $A$ be a uniform algebra on a compact Hausdorff space $X$, i.e. let $A$ be an algebra of continuous complex-valued functions on $X$, closed under uniform convergence on $X$, separating points and containing the constants. Let $\mathcal{M}$ denote the maximal ideal space of $A$. Gelfand's theory gives that $X$ may be embedded in $\mathcal{M}$ as a closed subset and each $f$ in $A$ has a natural extension to $\mathcal{M}$ as a continuous function. Set $\|f\|=\max _{X}|f|$.

We consider the following interpolation problem: choose $n$ points $M_{1}, \ldots, M_{n}$ in $\mathcal{M}$. Put

$$
I=\left\{g \in A \mid g\left(M_{j}\right)=0,1 \leq j \leq n\right\},
$$

and form the quotient-algebra $A / I . A / I$ is a commutative Banach algebra which is algebraically isomorphic to $\mathbb{C}^{n}$ under coordinatewise multiplication. For $f \in A$, $[f]$ denotes the coset of $f$ in $A / I$ and $\|[f]\|$ denotes the quotient norm. We put, for $w=\left(w_{1}, \ldots, w_{n}\right)$ in $\mathbb{C}^{n}$,

$$
\mathcal{D}=\left\{w \in \mathbb{C}^{n} \mid \exists f \in A \text { such that } f\left(M_{j}\right)=w_{j}, 1 \leq j \leq n \text {, and }\|[f]\| \leq 1\right\} .
$$

Our problem is to describe $\mathcal{D}$. It is easy to see that $\mathcal{D}$ is a closed subset of the closed unit polydisk $\Delta^{n}$ in $\mathbb{C}^{n}$ and has non-void interior. It turns out that $\mathcal{D}$ has the following property which we call hyperconvexity. We write \|\|$_{\Delta^{k}}$ for the supremum norm on $\Delta^{k}$. Let $P$ be a polynomial in $k$ variables and choose $k$ points $w^{\prime}, w^{\prime \prime}, \ldots, w^{(k)}$ in $\mathbb{C}^{n}$. We apply $P$ to this $k$-tuple of points, using the algebra structure in $\mathbb{C}^{n}$. Then

$$
P\left(w^{\prime}, w^{\prime \prime}, \ldots, w^{(k)}\right)=\left(P\left(w_{1}^{\prime}, w_{1}^{\prime \prime}, \ldots, w_{1}^{(k)}\right), P\left(w_{2}^{\prime}, w_{2}^{\prime \prime}, \ldots, w_{2}^{(k)}\right), \ldots\right) \in \mathbb{C}^{n} .
$$

1991 Mathematics Subject Classification: 32E30, 46J15.

The paper is in final form and no version of it will be published elsewhere. 
Definition. A compact set $Y$ in $\mathbb{C}^{n}$ with non-void interior is hyperconvex if whenever $w^{\prime}, w^{\prime \prime}, \ldots, w^{(k)}$ is a set of points in $Y$, then for every polynomial $P$ in $k$ variables with $\|P\|_{\Delta^{n}} \leq 1, P\left(w^{\prime}, w^{\prime \prime}, \ldots, w^{(k)}\right)$ again lies in $Y$.

TheOREM 1. For each uniform algebra $A$ and points $M_{1}, \ldots, M_{n}$, the set $\mathcal{D}$ is hyperconvex. Conversely, every hyperconvex set arises in this way from some $A, M_{1}, \ldots, M_{n}$.

Examples of hyperconvex sets occur in the 1916 work of G. Pick [6]. Pick was the first to consider interpolation problems of this type. He fixed an $n$-tuple of points $z_{1}, \ldots, z_{n}$ in the unit disk $|z|<1$.

Let us denote by $\mathcal{D}_{z}$ the set of all points $w=\left(w_{1}, \ldots, w_{n}\right)$ in $\mathbb{C}^{n}$ such that there exists a function $f$ in $H^{\infty}$ with $\|f\|_{\infty} \leq 1$ and $f\left(z_{j}\right)=w_{j}, 1 \leq j \leq n$.

PiCK's TheOrem. Let $w \in \mathbb{C}^{n}$. Then $w \in \mathcal{D}_{z}$ if and only if the matrix

$$
\left(\frac{1-w_{j} \bar{w}_{k}}{1-z_{j} \bar{z}_{k}}\right)
$$

is positive semi-definite.

We call the set $\mathcal{D}_{z}$ a Pick body. If $A$ is the disk algebra, then $\mathcal{M}$ is the closed unit disk and we may take the points $M_{j}$ to be $z_{j}, 1 \leq j \leq n$. It is easy to show that the associated set $\mathcal{D}$ coincides with the Pick body $\mathcal{D}_{z}$. In particular, $\mathcal{D}_{z}$ is a hyperconvex set in $\mathbb{C}^{n}$.

We have not found a geometric condition describing the general hyperconvex set, but we have obtained information in two special cases.

THEOREM 2. Each hyperconvex set $Y$ in $\mathbb{C}^{2}$ is either the bidisk $\Delta^{2}$ or is a Pick body $\mathcal{D}_{z}$ for some $\left(z_{1}, z_{2}\right)$. In either case there exists $\lambda, 0<\lambda \leq 1$, such that

$$
Y=\left\{\left(w_{1}, w_{2}\right)|| w_{1}|\leq 1,| w_{2}|\leq 1,| \frac{w_{1}-w_{2}}{1-\bar{w}_{1} w_{2}} \mid \leq \lambda\right\} .
$$

TheOrem 3. Fix n. A compact set $Y$ with non-void interior in $\mathbb{C}^{n}$ is a Pick body if and only if $Y$ is hyperconvex and $\exists z=\left(z_{1}, \ldots, z_{n}\right)$ on the boundary of $Y$ such that the powers $z, z^{2}, \ldots, z^{n-1}$ taken in the algebra $\mathbb{C}^{n}$ all lie on the boundary of $Y$, and $\left|z_{j}\right|<1$ for each $j$.

Theorem 2 is proved in [1] and Theorem 3 is proved in [2].

In addition, we have generalized Pick's theorem to an arbitrary uniform algebra $A$ and points $M_{1}, \ldots, M_{n}$, by giving a necessary and sufficient condition on a point $w$ to belong to $\mathcal{D}$ in terms of the positive semi-definiteness of a certain family of $n \times n$ matrices. (In Pick's case, where the algebra was the disk algebra, a single such condition sufficed.) (See [1], and also Nakazi [5] for related results.)

The interpolation problem we are considering is closely related to the so-called von Neumann inequality for operators on Hilbert space. 
The first connection between Pick interpolation and operator theory was made in the pioneering paper of Sarason [7]. Recent work in this area is contained in [1], in Lotto [3], and in Lotto and Steger [4].

\section{References}

[1] B. Cole, K. Lewis and J. Wermer, Pick conditions on a uniform algebra and von Neumann inequalities, J. Funct. Anal. 107 (1992), 235-254.

[2] - - - - A characterisation of Pick bodies, J. London Math. Soc. 48 (1993), 316-328.

[3] B. A. Lotto, von Neumann's inequality for commuting diagonalizable projections, preprint, 1991.

[4] B. A. Lotto and T. Steger, von Neumann's inequality fails for three commuting diagonalizable contractions, preprint, 1991.

[5] T. Nakazi, Commuting dilations and uniform algebras, Canad. J. Math. 42 (1990), 776789.

[6] G. Pick, Über die Beschränkungen analytischer Funktionen, welche durch vorgegebene Funktionswerte bewirkt werden, Math. Ann. 77 (1916), 7-23.

[7] D. Sarason, Generalized interpolation in $H^{\infty}$, Trans. Amer. Math. Soc. 127 (1967), 179-203. 